\title{
DISSIMILARIDADE GENÉTICA ENTRE FAMÍLIAS DE MELANCIA
}

Luniara Bastos Santos ${ }^{1}$, Aline Torquato Tavares²*, Tiago Alves Ferreira ${ }^{3}$, Gil Rodrigues Santos ${ }^{4}$, Renato Almeida Sarmento ${ }^{4}$, Danilo Alves Porto da Silva Lopes ${ }^{3}$, Ildon Rodrigues Nascimento ${ }^{4}$

\begin{abstract}
RESUMO - O melhoramento genético de uma espécie é dependente da amplitude da base genética disponível. Objetivou-se avaliar a dissimilaridade genética de famílias de melancia tipo Crimson Sweet obtidas do retrocruzamento do acesso resistente PI 595201 com Crimson Sweet. Foram utilizados 25 tratamentos sendo eles: 23 famílias obtidas de quatro retrocruzamentos do acesso PI 595201 (Genitor não-recorrente) com a cultivar Crimson Sweet (Genitor recorrente), selecionadas para resistência a PRSV-W e WMV e duas cultivares comerciais do tipo Crimson Sweet. Foram avaliadas 13 características para o estudo da similaridade genética e os componentes principais. Há variação genética entre os genótipos avaliado com formação de dez grupos distintos. As características que mais contribuíram para a dissimilaridade genética foram coloração da polpa (18,87\%), sólidos solúveis $(17,04 \%), \mathrm{pH}$ $(11,55 \%)$ e acidez titulável (9,98\%). Os genótipos mais dissimilares foram a cultivar Crimson Sweet ${ }^{\circledR}$ - Sakata e as ffamílias WMX-001G-14-02-55-01pl\#10, WMX-001G-14-02-55-01pl\#05 e WMX-001G09-04-58-07pl\#08.
\end{abstract}

Palavras chave: Citrullus lanatus (Thunb.) Matsum. \& Nakai, melhoramento genético, multivariada, potyvirus.

\section{GENETIC DISSIMILARITY AMONG WATERMELON FAMILIES}

\begin{abstract}
The genetic improvement of a species is dependent on the amplitude of the available genetic basis. The objective of this study was to evaluate the genetic dissimilarity of Crimson Sweet type watermelon families obtained from backcrossing of resistant access PI 595201 with Crimson Sweet. Twenty-five treatments were used: 23 families obtained from four backcrosses of the non-recurrent PI 595201 (Genitor nonrecurrent) cultivar Crimson Sweet (Recurrent Genitor), selected for resistance to PRSV-W and WMV and two commercial cultivars of the Crimson Sweet type. We evaluated 13 characteristics for the study of genetic similarity and the main components. There is genetic variation among genotypes evaluated with formation of ten distinct groups. The characteristics that contributed most to genetic dissimilarity were pulp (18.87\%), soluble solids (17.04\%), pH (11.55\%) and titratable acidity (9.98\%). The most dissimilar genotypes were Crimson Sweet ${ }^{\circledR}$ - Sakata and the families WMX-001G-14-02-55-01pl \# 10, WMX-001G14-02-55-01pl \# 05 and WMX-001G-09-04-58-07pl \# 08.
\end{abstract}

Keywords: Citrullus lanatus (Thunb.) Matsum. \& Nakai, genetic breeding, multivariate, potyvirus.

\footnotetext{
${ }^{1}$ Mestre em Produção Vegetal pela Universidade Federal do Tocantins - UFT.

2* Pós-doutoranda do Programa de Pós-Graduação em Produção Vegetal na Universidade Federal do Tocantins - UFT. alinet4t@hotmail.com *Autor para correspondência.

${ }^{3}$ Discente do Programa de Pós-Graduação em Produção Vegetal na Universidade Federal do Tocantins - UFT.

${ }^{4}$ Docente do curso de Agronomia, Universidade Federal do Tocantins - UFT, Campus de Gurupi-TO, Rua Badejós, lote. 7 , s/n, chácaras 69-72, Zona Rural. Cx. Postal 66. CEP 77402-970.
} 


\section{INTRODUÇÃO}

A melancia (Citrullus lanatus (Thunb.) Matsum. \& Nakai) é uma das mais importantes hortaliças cultivadas no Brasil. O cultivo é realizado em todas as regiões, destacando-se nos últimos anos as regiões CentroOeste, Norte e Nordeste do país onde se encontram expressivas áreas de produção no período do outono/ inverno (Furlaneto \& Bertani, 2015).

A ocorrência de doenças é um dos fatores que mais onera o custo de produção, destacando-se a ocorrência de viroses que reduzem substancialmente a produtividade e qualidade dos frutos colhidos (Vieira et al., 2005; Tavares et al., 2014).

Entre as espécies de vírus que acometem a cultura, está o Papaya ringspot virus strain watermelon (PRSVW), do gênero Potyvirus que causam sintomas muito severos como deformações foliares, bolhosidade e necrose (Aguiar et al., 2015; Tavares et al., 2014).

No Brasil, reação do tipo resistência genética a isolados brasileiros de PRSV-W foi identificado em C. lanatus var. lanatus em acessos da Nigéria (PI 595203 e PI 595201) (Azevedo et al., 2012).

A partir do cruzamento do acesso PI 595201 com cultivares de frutos do tipo Crimson Sweet, foram obtidas famílias avançadas de melancia com características semelhantes ao padrão comercial Crimson Sweet, que podem ser utilizadas na obtenção de novos híbridos, com maior adaptação às condições de cultivo, em comparação com as cultivares em uso que foram desenvolvidas em locais diferentes de onde serão recomendadas (Tavares et al., 2017).

Em um programa de melhoramento um dos pontos principais é a escolha dos pais para obter populações onde será realizada a seleção. Nesse contexto, recomendase a que seleção dos pais esteja aliada com a variabilidade genética ampla para características de interesse. Para facilitar o processo de seleção dos genótipos superiores e divergentes, uma das alternativas utilizadas é a estimativa da dissimilaridade genética entre as populações (Ferreira et al., 2003).

A dissimilaridade genética tem sido avaliada com o objetivo de identificar genótipos divergentes, que associados a média elevada poderão ser utilizados com objetivo de selecionar os genitores superiores que serão utilizados na formação da nova população (Cruz et al., 1994). Existem várias metodologias que podem ser utilizadas para avaliação da dissimilaridade genética entre os progenitores, entre as quais a análise por componentes principais tem se destacado (Cruz \& Regazzi, 2001).

Diversas medidas de similaridade têm sido propostas para a quantificação das distâncias entre genótipos, sendo, contudo, a distância generalizada de Mahalanobis a mais amplamente utilizada quando se dispõe de experimentos com repetições. Esta se diferencia das demais técnicas por levar em consideração as correlações residuais entre os caracteres avaliados (Cruz et al., 2004).

Entre os métodos de agrupamento, o método de otimização de Tocher tem sido um dos mais utilizados, pois, os grupos originalmente avaliados são subdivididos de forma que a maior distância média intragrupo seja menor que a distância intergrupo (Cruz et al., 1994), diminuindo o efeito grupos originalmente formados por características discrepantes.

O trabalho teve como objetivo mensurar a dissimilaridade genética entre famílias de melancia tipo Crimson Sweet obtidas do retrocruzamentos do acesso africano PI 595201 com Crimson Sweet.

\section{MATERIALE MÉTODOS}

O experimento foi conduzido na área experimental da Universidade Federal do Tocantins - UFT, Campus Universitário de Gurupi, localizado na região sul do Estado do Tocantins no ano agrícola de 2009. A temperatura média anual é de $29,5^{\circ} \mathrm{C}$, com precipitação anual média de $1.804 \mathrm{~mm}$.

O delineamento experimental utilizado foi o látice simples $(5 \times 5)$ com três repetições. Cada parcela foi composta por seis plantas (espaçadas 1,5 m entre plantas nas linhas e 2,0 m entre linhas de plantio) sendo considerada a parcela útil as quatro plantas centrais.

As mudas foram obtidas pela semeadura em copos descartáveis de $80 \mathrm{ml}$ contendo substrato comercial. O transplantio para o local definitivo foi feito 10 dias após semeadura, sendo o experimento implantado sob sistema de cultivo convencional. A calagem e as adubações foram realizadas de acordo com a análise de solo e a exigência da cultura.

Foram utilizados 25 tratamentos sendo eles: 23 famílias segregantes obtidas de retrocruzamentos do 
acesso PI 595201 (Genitor não-recorrente resistente) com a cultivar Crimson Sweet (Genitor recorrente suscetível), selecionadas para resistência a PRSV-W e WMV e duas cultivares comerciais do tipo Crimson Sweet. As famílias avaliadas foram: 1 - WMX-001G09-04-58-07pl\#07, 2 - WMX-001G-09-04-58-07pl\#08, 3 - WMX-001G-09-04-58-07pl\#14, 4 -WMX-001G-09-0403-03pl\#05, 5- WMX-001G-09-04-03-03pl\#06, 6 - WMX001G-09-04-03-03pl\#11, 7-WMX-001G-09-04-03-03pl\#12, 8 - WMX-001G-09-04-03-03pl\#13, 9 - WMX-001G-0904-03-03pl\#18, 10 - WMX-001G-09-04-03-03pl\#21, 11 -WMX-001G-09-04-03-03pl\#22, 12 - WMX-001G-14-0255-01pl\#01, 13-WMX-001G-14-02-55-01pl\#03, 14-WMX001G-14-02-55-01pl\#04, 15 - WMX-001G-14-02-5501pl\#05, 16 - WMX-001G-14-02-55-01pl\#07, 17 - WMX001G-14-02-55-01pl\#08, 18 - WMX-001G-14-02-5501pl\#09, 19 - WMX-001G-14-02-55-01pl\#10, 20 - WMX001G-14-02-55-01pl\#11, 21 - WMX-001G-14-02-5501pl\#12, 22 - WMX-001G-14-02-55-01pl\#13, 23 - WMX001G-14-02-55-01pl\#15, 24-Crimson Sweet e 25-Crimson Sweet - (Crimson Sweet ${ }^{\circledR}$ - Sakata).

Duas colheitas foram realizadas, a primeira 65 dias após o transplantio e segunda 5 dias após a primeira. As características avaliadas por parcela foram: Produtividade média total em $\mathrm{t} \mathrm{ha}^{-1}(\mathrm{PT})$; Massa média de frutos em kg (MMF); Formato do fruto (FF) obtido conforme índice proveniente da divisão do diâmetro transversal pelo diâmetro longitudinal, em que valores menores que 0,5 considerados frutos longos, entre 0,5 a 0,79 ovais e 0,80 a 1,00 frutos esféricos; Padrão de listras (PL), obtido por escala de notas, em que: 1 - frutos sem listas 2 - frutos com listra larga; 3 frutos com listra estreita; e 4 - frutos com mosqueado; Espessura da casca na região do pedúnculo em mm (ECP);Espessura da casca na região da inflorescência em cm (ECI); Espessura da casca na região distral em mm(ECD); Diâmetro do pedúnculo em mm(DP); Coloração externa (CE) conforme escala de nota em que: 1 - representa frutos verde escuro; 2 - frutos verde médio; 3 - frutos verde claro; e 4 - frutos amarelo, acidez titulável (AT); pH; sólidos solúveis (SS); coloração de polpa (CP), obtido por escala de notas, sendo: 1 - polpa vermelha; 2 - polpa rosa intenso; 3 - polpa rosa médio; 4 - polpa rosa claro; e 5 - polpa branca, conforme SILVA et al., (2006).

Os dados foram submetidos à análise de variância e as médias foram comparadas pelo teste de Scott \& Knott (1974) a 5\% de probabilidade. As medidas de dissimilaridade entre as famílias foram obtidas com as estimativas de médias para todas as características (Tabela 1) por meio da distância generalizada de Mahalanobis, com padronização dos dados. O agrupamento das famílias foi realizado utilizando-se o método de Tocher. A contribuição relativa das variáveis para a dissimilaridade genética foi feita utilizando o critério proposto por Singh (Cruz \& Regazzi, 2001). As análises foram realizadas por meio do aplicativo Genes (Cruz, 2001).

\section{RESULTADOS E DISCUSSÃO}

Para as características produtividade, massa média de frutos, espessura da casca na região do pedúnculo, espessura da casca na região da inflorescência, espessura da casca da região distal, diâmetro do pedúnculo, coloração externa, acidez titulável, $\mathrm{pH}$, sólidos solúveis e coloração da polpa houve a formação de grupos de distintos pelo teste de Skott-Knott o que denota existência de variabilidade genética (Tabelas 1 e 2). Já para as características formato de fruto, padrão de listras e $\mathrm{pH}$ não houve variação genética significativa (Tabelas 1 e 2).

A variabilidade é importante, pois ela diminui a vulnerabilidade genética, permite que indivíduos diferentes sejam formados dentro de uma mesma espécie, evitando sua extinção por algum fator adverso que possa ocorrer no meio em que elas estão expostas (Garcia et al., 2013).

A contribuição relativa das características para o agrupamento está apresentada na Tabela 3. Os quatro primeiros componentes principais explicaram 57,44\% da variação total (Tabela 3 ). As características que mais contribuíram para a dissimilaridade entre as famílias foram coloração da polpa (18,87\%), sólidos solúveis (17,04\%), pH (11,55\%) e acidez titulável (9,98\%), enquanto a que menos contribuiu foi a produção total $(0,04 \%)$. Esses resultados corroboram com os encontrados por Souza et al., (2005) onde os componentes que mais contribuíram para a dissimilaridade e famílias de melancia foram o teor de sólidos solúveis e o diâmetro longitudinal.

Pode-se observar que as características qualitativas foram as mais responsivas nos processos seletivos entre as famílias de melancia sendo no caso, os mais indicados para seleção das famílias mais divergentes.

Com relação aos componentes principais a coloração, teor de sólidos solúveis, pH, acidez titulável, coloração 
Tabela 1 - Médias para produtividade (PT em ton ha-1), massa média de frutos ( $\mathrm{g}^{-1}$ fruto ${ }^{-1}$ ), formato de fruto (FF), padrão de listras (PL), espessura da casca na região do pedúnculo (ECP em mm) e espessura da casca na região da inflorescência (ECI em mm) em 25 famílias de melancia. UFT, Gurupi-TO, 2009

\begin{tabular}{|c|c|c|c|c|c|c|}
\hline Famílias & PT (t.ha $\left.{ }^{-1}\right)$ & MMF (Kg) & FF & PL & $\mathrm{ECP}(\mathrm{mm})$ & $\mathrm{ECI}(\mathrm{mm})$ \\
\hline 1 & $17,92 b$ & $3,97 b$ & $0,91 \mathrm{a}$ & $2,71 \mathrm{a}$ & $12,53 \mathrm{f}$ & $10,05 c$ \\
\hline 2 & $14,39 b$ & $3,89 b$ & $0,90 a$ & $2,76 a$ & $13,21 \mathrm{f}$ & $7,89 d$ \\
\hline 3 & $11,38 b$ & $4,15 b$ & $0,94 a$ & $2,54 a$ & $14,23 \mathrm{e}$ & $8,41 d$ \\
\hline 4 & $17,2 b$ & $3,93 b$ & $0,96 a$ & $2,72 a$ & $12,87 f$ & $8,22 d$ \\
\hline 5 & $14,26 b$ & $3,60 \mathrm{~b}$ & $0,92 \mathrm{a}$ & $2,52 \mathrm{a}$ & $12,01 \mathrm{~g}$ & $9,41 \mathrm{c}$ \\
\hline 6 & $17,36 b$ & $4,33 b$ & $0,93 \mathrm{a}$ & $2,79 a$ & $11,83 \mathrm{~g}$ & $10,29 b$ \\
\hline 7 & $14,91 b$ & $4,01 b$ & $0,92 \mathrm{a}$ & $2,78 a$ & $14,89 \mathrm{e}$ & $11,01 b$ \\
\hline 8 & $19,48 b$ & $4,03 \mathrm{~b}$ & $0,93 a$ & $2,62 a$ & $14,36 \mathrm{e}$ & $8,73 d$ \\
\hline 9 & $30,40 a$ & $5,04 \mathrm{~b}$ & $0,94 a$ & $2,64 a$ & $14,51 \mathrm{e}$ & $10,42 b$ \\
\hline 10 & $12,21 b$ & $3,94 b$ & $0,95 \mathrm{a}$ & $2,89 a$ & $14,41 \mathrm{e}$ & $11,70 \mathrm{a}$ \\
\hline 11 & $14,10 \mathrm{~b}$ & $4,03 b$ & $0,94 a$ & $2,74 a$ & $12,70 \mathrm{f}$ & $10,01 c$ \\
\hline 12 & $29,48 a$ & $6,86 \mathrm{a}$ & $0,94 \mathrm{a}$ & $2,99 a$ & $17,14 \mathrm{~d}$ & $11,97 \mathrm{a}$ \\
\hline 13 & $22,14 b$ & $6,47 a$ & $0,95 a$ & $3,03 a$ & $16,01 d$ & $9,77 \mathrm{c}$ \\
\hline 14 & $13,28 b$ & $6,87 a$ & $0,91 \mathrm{a}$ & $2,00 \mathrm{~b}$ & $14,02 \mathrm{e}$ & $8,96 \mathrm{~d}$ \\
\hline 15 & $30,59 a$ & $7,11 \mathrm{a}$ & $0,92 \mathrm{a}$ & $2,90 \mathrm{a}$ & $15,82 d$ & $9,79 c$ \\
\hline 16 & $21,17 b$ & $6,22 a$ & $0,92 \mathrm{a}$ & $2,75 a$ & $15,46 \mathrm{e}$ & $10,96 b$ \\
\hline 17 & $34,17 \mathrm{a}$ & $6,55 a$ & $0,94 a$ & $2,80 a$ & $16,48 d$ & $11,37 \mathrm{a}$ \\
\hline 18 & $45,27 a$ & $7,47 a$ & $0,93 a$ & $2,91 \mathrm{a}$ & $19,18 b$ & $12,04 \mathrm{a}$ \\
\hline 19 & $25,45 a$ & $5,65 a$ & $0,94 \mathrm{a}$ & $2,80 \mathrm{a}$ & $17,79 \mathrm{c}$ & $8,75 d$ \\
\hline 20 & $29,27 a$ & $6,15 a$ & $0,96 \mathrm{a}$ & $2,87 a$ & $16,22 d$ & $10,74 \mathrm{~b}$ \\
\hline 21 & $27,16 a$ & $6,64 a$ & $0,93 a$ & $2,96 a$ & $18,19 \mathrm{c}$ & $12,00 \mathrm{a}$ \\
\hline 22 & $34,13 a$ & $7,05 b$ & $0,96 \mathrm{a}$ & $2,66 a$ & $17,87 \mathrm{c}$ & $10,47 b$ \\
\hline 23 & $15,16 b$ & $4,17 b$ & $0,92 \mathrm{a}$ & $2,88 a$ & $11,46 \mathrm{~g}$ & $9,88 c$ \\
\hline 24 & $35,28 a$ & $7,52 \mathrm{a}$ & $0,92 \mathrm{a}$ & $2,84 a$ & $20,91 \mathrm{a}$ & $9,61 \mathrm{c}$ \\
\hline 25 & $31,01 \mathrm{a}$ & $6,80 a$ & $0,92 \mathrm{a}$ & $2,63 a$ & $19,11 b$ & $10,31 b$ \\
\hline
\end{tabular}

Médias seguidas da mesma letra não diferem entre si pelo teste de Scott-Knott a 5\% de probabilidade. Produtividade média total (PT), Massa média de frutos (MMF), formato do fruto (FF), Padrão de listras (PL), espessura de casca região do pedúnculo em mm (ECP); espessura de casca da região de inflorescência em mm (ECI).

externa e diâmetro do pedúnculo são caracteres de importância para o melhoramento e responderam por $72,76 \%$ da variação entre as famílias. Estes resultados não estão de acordo com Cruz \& Regazzi (2001), que recomendam $70 \%$ ou mais da variância total para os dois primeiros componentes principais.

A identificação dos grupos realizada pelo método de agrupamento proposto por Tocher possibilitou a divisão das 25 famílias em dez grupos (Tabela 4). É esperado que as famílias pertencentes ao mesmo grupo apresentem maior similaridade entre si e dissimilaridade genética entre grupos.

As duas cultivares Crimson Sweet comercias utilizadas como testemunhas ficaram em grupos amplamente distintos (Tabela 4, Figura 1) e as famílias em grupos intermediários a elas. Isso mostra que as famílias são pouco divergentes e se assemelharam com as cultivares comerciais, pois, apesar de estarem em grupos distintos a distância entre os grupos é pequena como pode ser observado na (Figura 1). Os acessos foram originados de retrocruzamentos com a cultivar Crimson Sweet comercial para incorporação das características de interesse, o que pode ter favorecido para a formação de muitos grupos, porém próximos.

A escolha dos genitores depende de vários fatores, tais como: dos caracteres a serem melhorados, do tipo de herança e da fonte de germoplasma disponível (Fehr, 1987), de forma que os cruzamentos são realizados entre genitores fenotipicamente complementares e portadores dos caracteres necessários para atender os objetivos do programa de melhoramento em questão (Borém \& Miranda, 2009). Nesse sentido, o cruzamento de famílias de grupos distintos com características de alta produtividade com famílias de características físico- 
Tabela 2 - Médias para espessura da casca da região distal (ECD em mm), diâmetro do pedúnculo (DP em mm), coloração externa, acidez titulável (AT), pH (PH), sólidos solúveis (SS) e coloração da polpa (CP) em 25 famílias de melancia. UFT, Gurupi-TO, 2009

\begin{tabular}{|c|c|c|c|c|c|c|c|}
\hline Famílias & $\mathrm{ECD}(\mathrm{mm})$ & $\mathrm{DP}(\mathrm{mm})$ & CE & AT & $\mathrm{PH}$ & SS & CP \\
\hline 1 & $13,63 d$ & $18,51 \mathrm{e}$ & $2,20 a$ & $0,08 b$ & $4,32 \mathrm{a}$ & $6,61 b$ & 3,39a \\
\hline 2 & $13,61 d$ & $18,96 \mathrm{e}$ & $1,63 b$ & $0,09 b$ & $5,02 \mathrm{a}$ & $7,21 \mathrm{a}$ & $2,26 b$ \\
\hline 3 & $13,92 d$ & $20,66 c$ & $2,28 a$ & $0,09 b$ & $5,05 a$ & $5,43 b$ & $1,84 b$ \\
\hline 4 & $13,37 d$ & $18,43 \mathrm{e}$ & $2,09 a$ & $0,09 b$ & $4,99 a$ & $6,24 b$ & $2,15 b$ \\
\hline 5 & $11,66 \mathrm{e}$ & 18,85 e & $2,01 \mathrm{a}$ & $0,09 b$ & $4,97 a$ & $6,52 b$ & $2,23 b$ \\
\hline 6 & $14,91 \mathrm{c}$ & $19,70 d$ & $1,64 b$ & $0,08 b$ & $5,03 a$ & $7,35 \mathrm{a}$ & $1,71 b$ \\
\hline 7 & $14,14 \mathrm{c}$ & $21,36 \mathrm{c}$ & $2,13 a$ & $0,10 b$ & $4,97 a$ & $7,20 \mathrm{a}$ & $1,89 b$ \\
\hline 8 & $14,67 \mathrm{c}$ & $18,65 \mathrm{e}$ & $2,27 a$ & $0,08 b$ & $4,08 a$ & $6,42 b$ & $2,01 b$ \\
\hline 9 & $12,26 \mathrm{e}$ & $18,88 \mathrm{e}$ & $2,37 a$ & $0,07 b$ & $5,65 a$ & $7,31 \mathrm{a}$ & $2,16 b$ \\
\hline 10 & $14,19 \mathrm{c}$ & $19,21 \mathrm{e}$ & $1,92 \mathrm{a}$ & $0,08 b$ & $4,95 a$ & $6,79 b$ & $1,79 b$ \\
\hline 11 & $14,29 c$ & 18,89 e & $1,92 \mathrm{a}$ & $0,07 b$ & $5,62 \mathrm{a}$ & $6,68 b$ & $1,68 b$ \\
\hline 12 & $17,43 a$ & $18,58 \mathrm{e}$ & $2,03 a$ & $0,09 b$ & $4,91 \mathrm{a}$ & $7,76 a$ & $1,87 b$ \\
\hline 13 & $15,10 \mathrm{~b}$ & $17,47 \mathrm{f}$ & $2,77 a$ & $0,11 b$ & $3,85 a$ & $6,70 \mathrm{~b}$ & $2,54 \mathrm{a}$ \\
\hline 14 & $10,43 f$ & $21,10 \mathrm{c}$ & $1,38 b$ & $0,17 a$ & $4,92 \mathrm{a}$ & $6,74 b$ & $3,60 a$ \\
\hline 15 & $15,18 b$ & $18,26 \mathrm{e}$ & $1,99 a$ & $0,09 b$ & $4,73 a$ & 7,39a & $2,34 b$ \\
\hline 16 & $14,52 \mathrm{c}$ & $17,76 \mathrm{f}$ & $1,54 b$ & $0,11 b$ & $4,87 a$ & $6,16 b$ & $2,61 \mathrm{a}$ \\
\hline 17 & $16,65 a$ & 18,69 e & $1,80 \mathrm{~b}$ & $0,11 b$ & $5,57 a$ & $7,45 \mathrm{a}$ & $1,91 b$ \\
\hline 18 & $16,87 \mathrm{a}$ & $20,04 d$ & $1,99 a$ & $0,09 b$ & $5,49 a$ & $6,86 b$ & $2,87 \mathrm{a}$ \\
\hline 19 & $17,35 a$ & 18,87 e & $2,22 a$ & $0,08 b$ & $4,16 \mathrm{a}$ & $7,41 a$ & $2,51 a$ \\
\hline 20 & $16,77 \mathrm{a}$ & $18,46 \mathrm{e}$ & $1,34 b$ & $0,09 b$ & $4,91 \mathrm{a}$ & $7,19 a$ & $1,80 \mathrm{~b}$ \\
\hline 21 & $16,28 a$ & $20,04 d$ & $1,95 a$ & $0,08 b$ & $8,54 a$ & 7,69a & $2,64 a$ \\
\hline 22 & $16,79 a$ & $19,54 d$ & $1,61 b$ & $0,09 b$ & $5,52 \mathrm{a}$ & $7,12 \mathrm{a}$ & $2,56 a$ \\
\hline 23 & $14,79 \mathrm{c}$ & $16,36 \mathrm{~g}$ & $2,19 a$ & $0,07 b$ & $5,10 a$ & $6,98 a$ & $1,82 b$ \\
\hline 24 & $15,62 b$ & $24,14 \mathrm{a}$ & $2,07 a$ & $0,08 b$ & $5,62 \mathrm{a}$ & $7,71 \mathrm{a}$ & $2,00 \mathrm{~b}$ \\
\hline 25 & $15,86 b$ & $22,47 b$ & $1,72 b$ & $0,10 b$ & $4,97 a$ & $6,59 b$ & $1,67 b$ \\
\hline
\end{tabular}

Médias seguidas da mesma letra não diferem entre si pelo teste de Scott-Knott a 5\% de probabilidade. Espessura de casca da região distral em mm (ECD); diâmetro do pendúnculo em mm (DP); coloração externa (CE); acidez titulável (AT); pH (PH); sólidos solúveis (SS); coloração de polpa (CP).

Tabela 3 - Contribuição relativa de 13 características avaliadas em 25 famílias de melancia UFT, Gurupi-TO, 2009

\begin{tabular}{cc}
\hline Características & Contribuição relativa (\%) \\
\hline CP & 18,87 \\
SS & 17,04 \\
PH & 11,55 \\
AT & 9,98 \\
CE & 8,92 \\
DP & 7,10 \\
ECD & 6,63 \\
ECI & 5,81 \\
ECP & 5,32 \\
PL & 4,33 \\
FF & 4,21 \\
MMF & 0,11 \\
P T & 0,04 \\
\hline
\end{tabular}

${ }^{1}$ Produtividade média total (PT), massa média do fruto (MMF) formato do fruto (FF); Padrão de listras (PL); espessura da casca na região do pedúnculo (ECP); espessura da casca na região da inflorescência (ECI); espessura da casca na região distral (ECD); diâmetro do pedúnculo (DP); coloração externa (CE); acidez titulável (AT); pH (PH); sólidos solúveis (SS); coloração de polpa(CP).
Tabela 4 - Agrupamento das 25 famílias de melancia pelo método de Tocher. UFT, Gurupi-TO, 2009

\begin{tabular}{ccc}
\hline Agrupamento & Número de famílias & Famílias \\
\hline I & 3 & $10,24,11$ \\
II & 3 & $9,23,16$ \\
III & 6 & $6,20,13,7,12$ \\
IV & 4 & $4,18,17$ \\
V & 3 & $1,22,8$ \\
VI & 2 & 14,21 \\
VII & 1 & 2 \\
VIII & 1 & 15 \\
IX & 1 & 19 \\
X & 1 & 25 \\
\hline
\end{tabular}

químicas superiores, possuem potencial genético para estratégias futuras de seleção.

A classificação de genótipos utilizando os recursos multivariados é escassa na literatura para melancia. Entretanto 


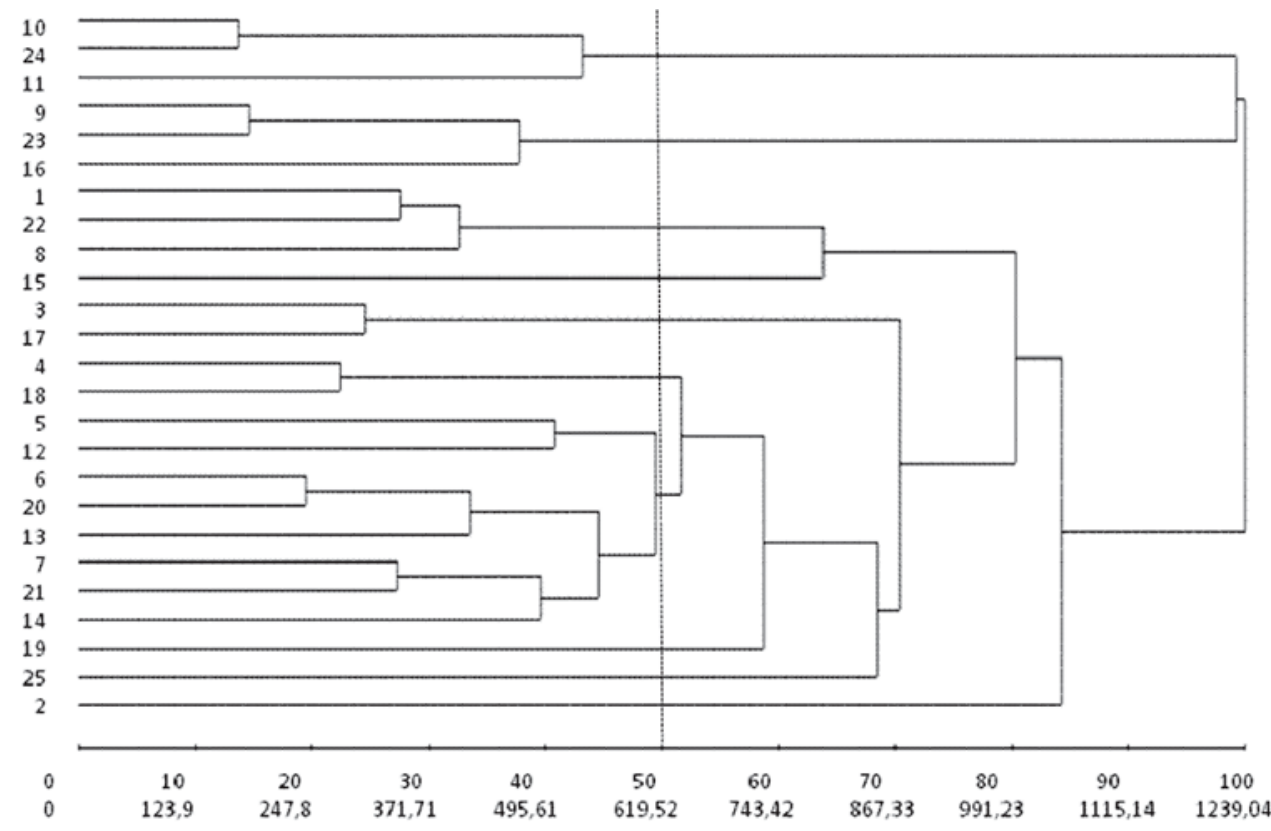

Figura 1 - Padrão de similaridade de 25 famílias de melancia para 13 características fenotípicas com base na distância de Mahalanobis (método do vizinho mais próximo). UFT, Gurupi-TO, 2009

essa metodologia têm oferecido contribuições efetivas na discriminação de genótipos potencialmente utilizáveis no melhoramento genético de várias culturas, inclusive com indicação das características que potencialmente auxiliam na obtenção de populações geneticamente divergentes (Santos et al., 2000; Kvitschal, 2008).

Espera-se na metodologia multivariada que o grau de parentesco e a dissimilaridade genética forneçam informações sobre o grau de complementaridade entre os genitores envolvidos nos cruzamentos, assim como o grau de variação genética nas populações segregantes (Kvitschal, 2008). Quando comparado ao método de Tocher, o hierárquico do "vizinho mais próximo" (Figura 1) revelou resultado semelhante, sendo formado o mesmo número de grupos e, em cada grupo, os mesmos cultivares. Concordância na discriminação de grupos entre esses dois métodos também foi verificada por Vidigal et al., (1997), com cultivares de mandioca e por Amaral Júnior (1994), com acessos de moranga (Cucurbita maxima).

\section{CONCLUSÕES}

A identificação dos grupos realizada pelo método de agrupamento proposto por Tocher possibilitou a divisão das 25 famílias em dez grupos.
As duas cultivares Crimson Sweet avaliadas ficaram em grupos amplamente distintos e as famílias em grupos intermediários a elas.

Em geral as famílias são pouco divergentes e se assemelharam com as cultivares comerciais, pois, apesar de estarem em grupos distintos a distância entre os grupos é pequena.

Os genótipos mais divergentes foram a cultivar Crimson Sweet ${ }^{\circledR}$ - Sakata e as famílias WMX-001G1402-55-01pl\#10, WMX-001G-14-02-55-01pl\#05 e WMX001G-09-04-58-07pl\#08.

\section{LITERATURA CITADA}

AGUIAR, R.W.S.; RODRIGUES, A.; PORTELLA, A.C.F. et al. Serological Identification of Virus in Watermelon Production Fields in the Tocantins State. Brazilian Archives of Biology and Technology, v.1, n.2, p.1-6, 2015.

AMARAL JÚNIOR, A.T. Análise multivariada e isoenzimática da divergência genética entre acessos de moranga (Cucurbita maxima Duchesne).

Dissertação (Mestrado em Genética e Melhoramento). Viçosa-MG: UGV, 1994. 95p. 
AZEVEDO, S.M.; MALUF, W.R.; FARIA, M.V. et al. Inheritance of resistance to the Papaya ringspot virus-watermelon strain (PRSV-W) from watermelon accession 'PI 595201'. Crop Breeding and Applied Biotechnology, Viçosa, v.12, n.1, p.67-75, 2012.

BORÉM, A.; MIRANDA, G.V. Melhoramento de plantas. 5.ed. Viçosa: UFV, 2009. 529p.

CRUZ, C.D. Programa genes: aplicativo computacional em genética e estatística. Viçosa: UFV, 2001. 648p.

CRUZ, C.D.; REGAZZI, A.J. Modelos biométricos aplicados ao melhoramento genético. UFV, 3. (eds.), Viçosa, 2001. 390p.

CRUZ, C.D.; VENCOVSKY, R.; CARVALHO, S.P. Estudo sobre divergência genética III. Comparação de técnicas multivariadas. Revista Ceres, Viçosa, v.41, n.234, p.191-201, 1994.

CRUZ, C.D.; REGAZZI, A.J. Modelos biométricos aplicados ao melhoramento genético. Viçosa: UFV, Imprensa Universitária. 2001. 390 p.

CRUZ, C.D.; REGAZZI, A.J. CARNEIRO, P.C.S. Modelos biométricos aplicados ao melhoramento genético, v.1, 3 ed. Viçosa: UFV, 2004. 480p.

FEHR, W.R. Principles of cultivar development: theory and technique. New York: MacMillan. 1987. 536p.

FERREIRA, M.A.J.F.; QUEIRÓZ, M.A.; BRAZ, L.T. et al. Correlações genotípicas, fenotípicas e de ambiente entre dez caracteres de melancia e suas implicações para o melhoramento genético. Horticultura Brasileira, v.21, n.3, p.438-442. 2003.

FURLANETO, F.P.B.; BERTANI, R.M.A. Melancia Do Brasil para o mundo. Agência Paulista de Tecnologia dos Agronegócios (APTA) - Polo Centro-oeste. Revista Campo \& Negócios Hortifrúti, 2015. In: http://www.revistacampoenegocios.com.br/ melancia-do-brasil-para-o-mundo/. (acessado em 28 de Junho de 2017).

GARCIA, L.A.C.; PINTO, L.R.; LANDELL, M.G.A. Importância da manutenção de variabilidade genética para os produtores rurais. Pesquisa \& Tecnologia, v.10, n.2, jul-dez de 2013.
KVITSCHAL, M.V. Caracterização e divergência genética de germoplasma de mandioca-de-mesa da região urbana de Maringá, Paraná. Tese (Doutorado em Genética e Melhoramento). Universidade Estadual de Maringá - UEM, Maringá, 2008. 140p.

SANTOS, R.C.; MOREIRA, J.N.A.; FARIAS, R.H.; DUARTE, J.M. Classificação de genótipos de amendoim baseados nos descritores agromorfológicos e isoenzimáticos. Ciência Rural, v.30, n.1, p.55-59, 2000.

SCOTT, A.J.; KNOTT, M. A cluster analysis method for grouping means in the analysis of variance. Biometrics, v.30, p.507-512, 1974.

SILVA, M.L.; QUEIRÓZ, M.A.; FERREIRA, M.A.J.F. et al. Caracterização morfológica e molecular de acessos de melancia. Horticultura Brasileira, Brasília, v.24, n.4, p.405-409, 2006.

SOUZA, F.F.; QUEIRÓZ, M.A.; DIAS, R.S.C. Divergência genética em famílias de melancia. Horticultura Brasileira, Brasília, v.23, n.2, p.179183, 2005.

TAVARES, A.T.; CHAVES, P.P.N.; AGUIAR, R.W. et al. Reação fenotípica de plantas de abóbora e melão à infecção por isolados simples de ZYMV e misto de ZYMV+SQMV. Journal of Biotechnology and Biodiversity, v.5, n.1, p.79-87, 2014.

TAVARES, A.T.; FERREIRA, T.A.; ZANATTA, E.E. et al. Estabilidade e adaptabilidade de genótipos de melancia em várzea tropical. Cultura Agronômica, Ilha Solteira, v.26, n.3, p.362-374, 2017.

VIEIRA, J.V.; ÁVILA, A.C.; PINTO, M.N. et al. Avaliação da coleção de germoplasma de melancia da Embrapa hortaliças para tolerância a viroses. EMBRAPA, Brasília. Boletim de pesquisa e desenvolvimento, $1^{\text {a }}$ ed., 2005. 13p.

VIDIGAL, M.C.G.; VIDIGAL FILHO, P.S.; AMARAL JÚNIOR, A.T. et al. Divergência genética entre cultivares de mandioca por meio de estatística multivariada. Bragantia, v.56, n.2, p.45-51, 1997.

Recebido para publicação em 16/1/2018 e aprovado em 27/3/2018. 